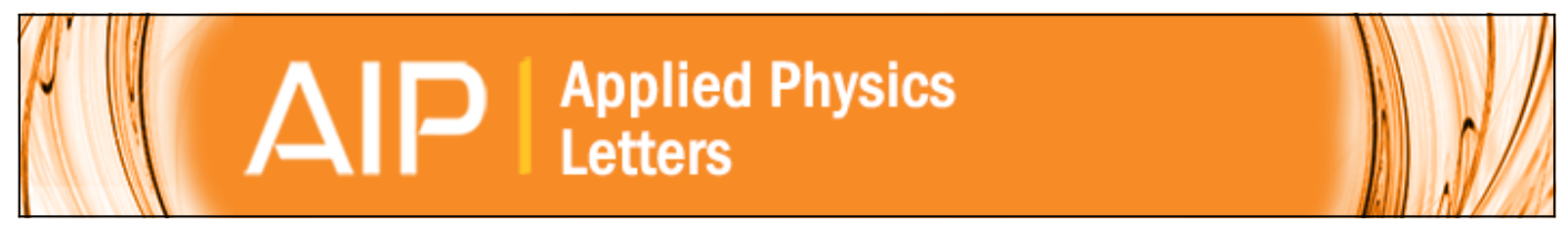

\title{
GaN-based waveguide devices for long-wavelength optical communications
}

R. Hui, S. Taherion, Y. Wan, J. Li, S. X. Jin, J. Y. Lin, and H. X. Jiang

Citation: Applied Physics Letters 82, 1326 (2003); doi: 10.1063/1.1557790

View online: http://dx.doi.org/10.1063/1.1557790

View Table of Contents: http://scitation.aip.org/content/aip/journal/apl/82/9?ver=pdfcov

Published by the AIP Publishing

Articles you may be interested in

Dispersion properties and low infrared optical losses in epitaxial AIN on sapphire substrate in the visible and infrared range

J. Appl. Phys. 115, 163515 (2014); 10.1063/1.4873236

Refractive index of III-metal-polar and N-polar AIGaN waveguides grown by metal organic chemical vapor deposition

Appl. Phys. Lett. 102, 221106 (2013); 10.1063/1.4800554

Birefringence of GaN/AIGaN optical waveguides

Appl. Phys. Lett. 83, 1698 (2003); 10.1063/1.1606103

Optical properties of low-pressure metalorganic vapor phase epitaxy Al x Ga 1-x N thin-film waveguides by prism coupling technique

Appl. Phys. Lett. 74, 3960 (1999); 10.1063/1.124236

Phase-matched optical second-harmonic generation in GaN and AIN slab waveguides

J. Appl. Phys. 85, 2497 (1999); 10.1063/1.369611

$M M R$

TECH NOLO GIES

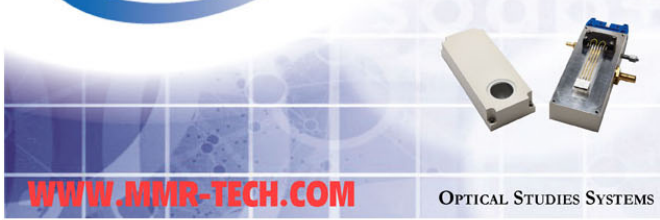

THE WORLD'S RESOURCE FOR VARIABLE TEMPERATURE SOLID STATE CHARACTERIZATION

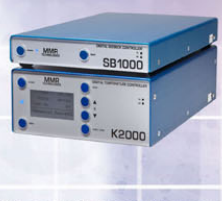

SeEbeck Studies Systems

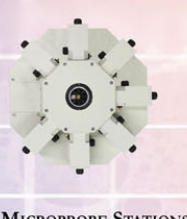

Microprobe Stations
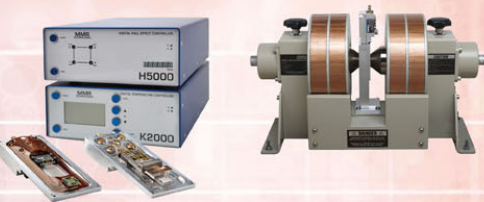

Hall EfFect Study Systems and Magnets 


\title{
GaN-based waveguide devices for long-wavelength optical communications
}

\author{
R. Hui, ${ }^{\text {a) }}$ S. Taherion, and Y. Wan \\ Department of Electrical Engineering and Computer Science, The University of Kansas, Lawrence, \\ Kansas 66044 \\ J. Li, S. X. Jin, J. Y. Lin, and H. X. Jiang \\ Department of Physics, Kansas State University, Manhattan, Kansas 66506-2601
}

(Received 7 November 2002; accepted 8 January 2003)

\begin{abstract}
Refractive indices of $\mathrm{Al}_{x} \mathrm{Ga}_{1-x} \mathrm{~N}$ with different $\mathrm{Al}$ concentrations have been measured in infrared wavelength regions. Single-mode ridged optical waveguide devices using GaN/AlGaN heterostructures have been designed, fabricated, and characterized for operation in $1550 \mathrm{~nm}$ wavelength window. The feasibility of developing photonic integrated circuits based on III-nitride wide-band-gap semiconductors for fiber-optical communications has been discussed. (C) 2003 American Institute of Physics. [DOI: 10.1063/1.1557790]
\end{abstract}

In order to make full use of the wide bandwidth provided by optical fibers, more and more wavelength channels have been used in wavelength division multiplexed (WDM) optical networks. Among others, WDM optical demultiplexers, optical switches, and wavelength routers are fundamental devices in multiwavelength, dynamic optical networks. With the rapid advancement of photonic integrated circuits (PIC), silica-based array waveguide grating (AWG) has become a popular approach for WDM multiplexing and demultiplexing. ${ }^{1}$ Since silica is not a semiconductor material and hence purely passive, the transfer function of a silicabased AWG is usually not tunable, or can only be slowly tuned by thermal effect. ${ }^{2,3}$ InP has been another popular material to make planar waveguide PICs. As a semiconductor material, InP-based PIC can potentially be made fast tunable with carrier injection, ${ }^{4}$ however, due to its high refractive index, high temperature sensitivity, and high insertion loss, ${ }^{5}$ InP-based AWG devices have so far not become commercially competitive.

III-nitride wide-band-gap semiconductor materials have attracted much attention in recent years. ${ }^{6,7}$ In addition to emitting in UV/blue wavelength region, III-nitride optoelectronic devices are able to operate at high temperatures and high power levels due to their mechanical hardness and larger band offsets. Research in III nitrides has so far been focused on their applications in blue/UV wavelength regions. Their optical characteristics and potential applications in infrared for optical communications remain largely unknown. In this letter, we propose to make functional optical waveguide devices using GaN/AlGaN semiconductor materials and explore their potential applications in infrared wavelength regions for fiber-optic communications.

In order to design guided-wave optical devices, the knowledge of material refractive indices in the operating wavelength region is essential. Due to the unavailability of experimental data in infrared, we have conducted the refractive index measurements for $\mathrm{Al}_{x} \mathrm{Ga}_{1-x} \mathrm{~N}$ with different $\mathrm{Al}$ molar fractions. In order to perform this measurement, a number of samples of $\mathrm{Al}_{x} \mathrm{Ga}_{1-x} \mathrm{~N}$ films were grown by met-

a)Electronic mail: hui@eecs.ukans.edu alorganic chemical vapor deposition (MOCVD) on sapphire substrates. The films thickness range from 1.1 to $1.5 \mu \mathrm{m}$ and Al molar fractions range from $x=0.1$ to $x=0.7{ }^{8}$ To evaluate the refractive index of each film, optical transmission spectra were measured. Due to the Fabry-Perot (FP) interference caused by the two facets of the film (one facet is between $\mathrm{Al}_{x} \mathrm{Ga}_{1-x} \mathrm{~N}$ and the air and the other facet is formed between $\mathrm{Al}_{x} \mathrm{Ga}_{1-x} \mathrm{~N}$ and sapphire), optical transmission efficiency is wavelength dependent. With the knowledge of the film thickness, the film refractive index can be obtained by best fitting the measured optical transmission spectrum to a well-known FP transmission equation.

Figure 1(a) shows the measured refractive indices of $\mathrm{Al}_{x} \mathrm{Ga}_{1-x} \mathrm{~N}$ versus wavelength for several different $\mathrm{Al}$ molar fractions. The continuous curves in the same figure were numerical fittings by using the first order Sellmeier dispersion formula:

$$
n(\lambda)=\sqrt{1+\frac{\left(B_{0}+B_{1} x+B_{2} x^{2}\right) \lambda^{2}}{\lambda^{2}-\left(C_{0}+C_{1} x\right)^{2}}} .
$$

The coefficients for best fitting are displayed in Fig. 1(a) and their variations versus Al molar fraction $x$ are shown in Fig. 1(b). Since we are mostly interested in the refractive indices in $1550 \mathrm{~nm}$ wavelength window, this information can be collected from Fig. 1 and the following polynomial expression is obtained for the $\mathrm{Al}$ molar fraction $(x)$ dependence of the refractive index at $1550 \mathrm{~nm}$ wavelength:

$$
n(1550 \mathrm{~nm})=0.431 x^{2}-0.735 x+2.335 .
$$

The monotonic decrease of $\mathrm{Al}_{x} \mathrm{Ga}_{1-x} \mathrm{~N}$ refractive index with the increase of Al molar fraction $x$ makes the design of single-mode optical waveguide devices straightforward.

We used beam-propagation method (BPM) simulation tools to design single-mode optical waveguide devices. Figure 2 shows schematically the cross section of the designed single-mode waveguide based on $\mathrm{GaN}$ core and $\mathrm{Al}_{x} \mathrm{Ga}_{1-{ }_{x} \mathrm{~N}}$ cladding. Several different waveguide configurations have been designed, including straight waveguides, and $2 \times 2$ waveguide couplers. To verify the design, a number of waveguide samples were prepared. In the fabrication process, a 4- $\mu$ m-thick epitaxial film of $\mathrm{Al}_{x} \mathrm{Ga}_{1-x} \mathrm{~N}$ was grown on a 

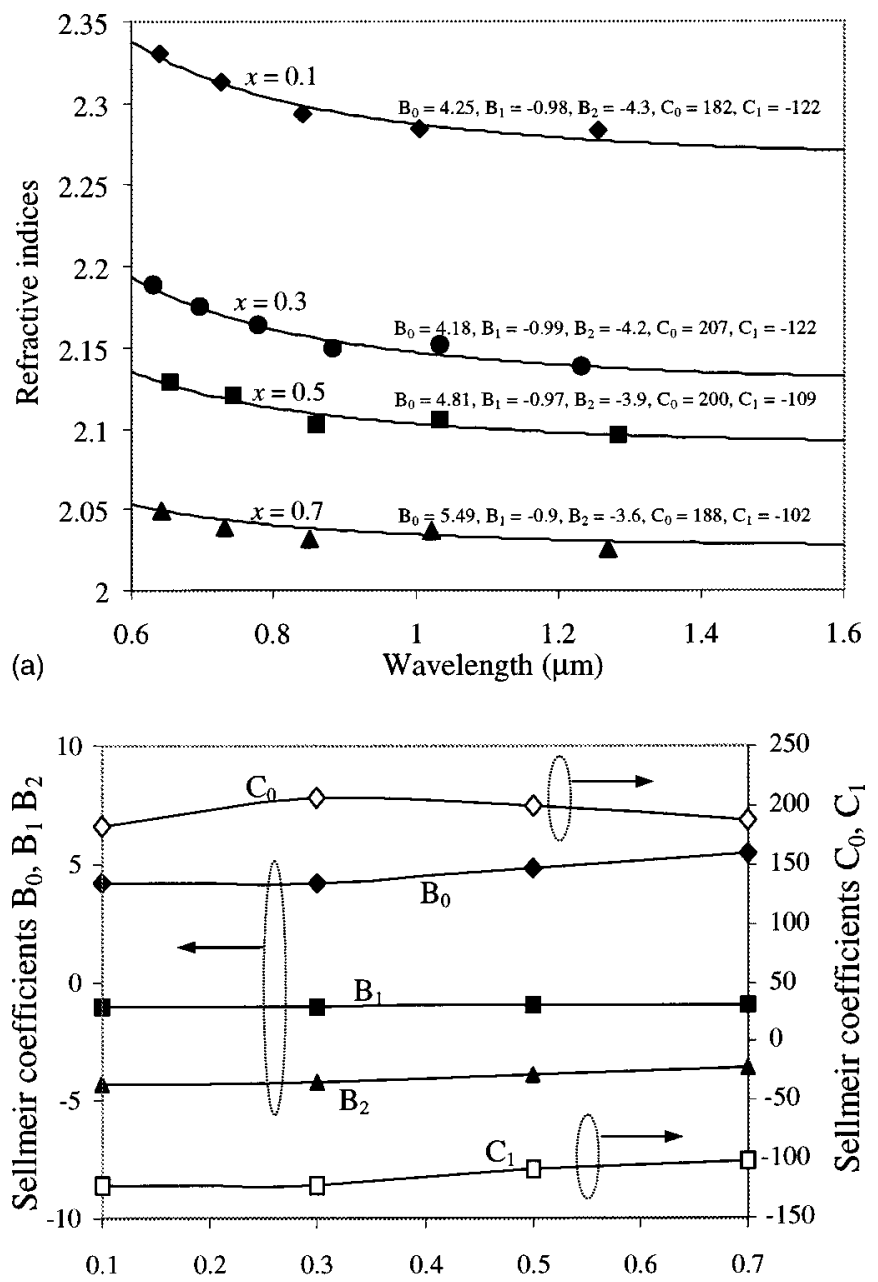

(b)

Al molar fraction $x$

FIG. 1. (a) Refractive indices of $\mathrm{Al}_{x} \mathrm{Ga}_{1-x} \mathrm{~N}$ vs wavelength for several different Al molar fractions. (b) Sellmeier expansion coefficients vs Al molar fractions.

sapphire substrate and a 3- $\mu$ m-thick GaN film was deposited on top of the $\mathrm{Al}_{x} \mathrm{Ga}_{1-x} \mathrm{~N}$ layer and then the optical waveguide structures were formed by photolithographic patterning and inductively coupled plasma (ICP) dry etching. ${ }^{9}$ According to the design, the etching depth is controlled at $\sim 2.8$ $\mu \mathrm{m}$ and the $\mathrm{Al}$ molar fraction $x$ is about $3 \%$. As an example,

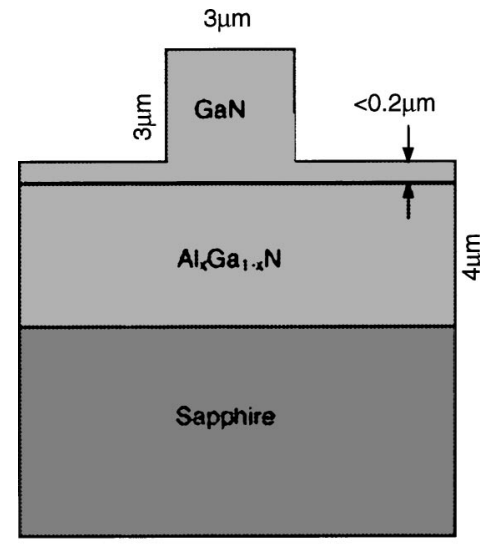

(a)

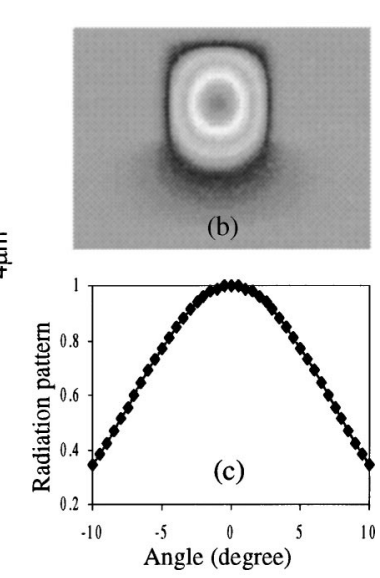

FIG. 2. Optical waveguide design using $\mathrm{GaN} / \mathrm{Al}_{x} \mathrm{Ga}_{1-x} \mathrm{~N}$ heterostructures grown on sapphire substrates: (a) waveguide cross section: (b) simulated single-mode structure; and (c) radiation pattern vs exit angle.
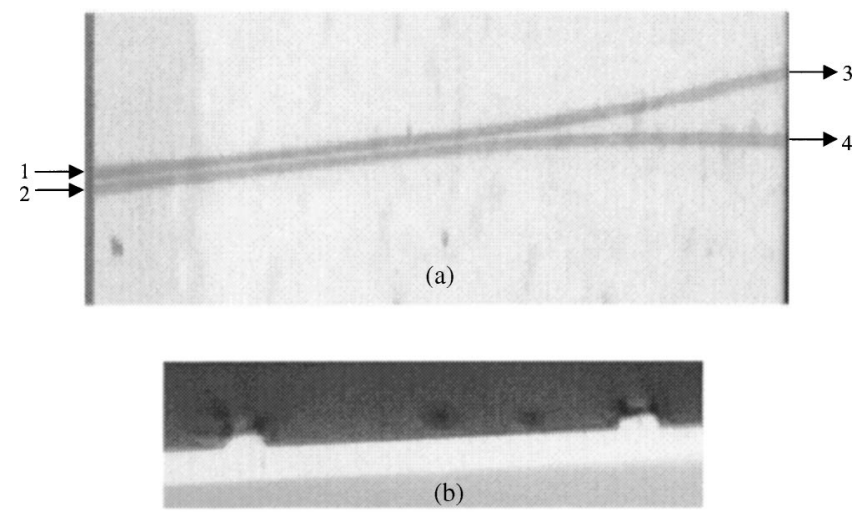

FIG. 3. Optical microscope images of a $2 \times 2 \mathrm{GaN} / \mathrm{AlGaN}$ heterostructure optical waveguide coupler (a) top view and (b) cross section at the output.

Fig. 3 shows a typical $2 \times 2$ waveguide coupler fabricated with this process. The power splitting ratio of this particular coupler was designed to be $3 \mathrm{~dB}$.

To characterize the waveguide samples, a fiber-optic setup operating in the $1550 \mathrm{~nm}$ wavelength region was used. Optical coupling at the input and the output of the waveguide was accomplished by using tapered single-mode fibers with 6- $\mu \mathrm{m}$-working distance and $2.5-\mu \mathrm{m}$-spot size of the focus. Each tapered fiber end was mounted on a five-dimensional precision positioning stage to optimize the optical coupling efficiency. A tunable laser diode was used as the light source and an optical power meter was used to measure the optical power that passes through the waveguide. Figure 4 shows the measured optical power versus the horizontal position of the tapered output fiber probe. In this measurement, the input fiber probe was aligned with one of the two input ports of the waveguide coupler. This figure clearly shows an $\sim 50 \%$ power splitting realized by this GaN/AlGaN waveguide coupler and thus validated our original design. In order to evaluate the loss of the waveguide, we have measured a 1.395$\mathrm{mm}$-long straight waveguide. Although the total fiber-tochip-to-fiber loss is $\sim 13 \mathrm{~dB}$, the coupling loss between fiber and waveguide is a major contributor. Our experiment was based on the measurement of FP interference caused by the

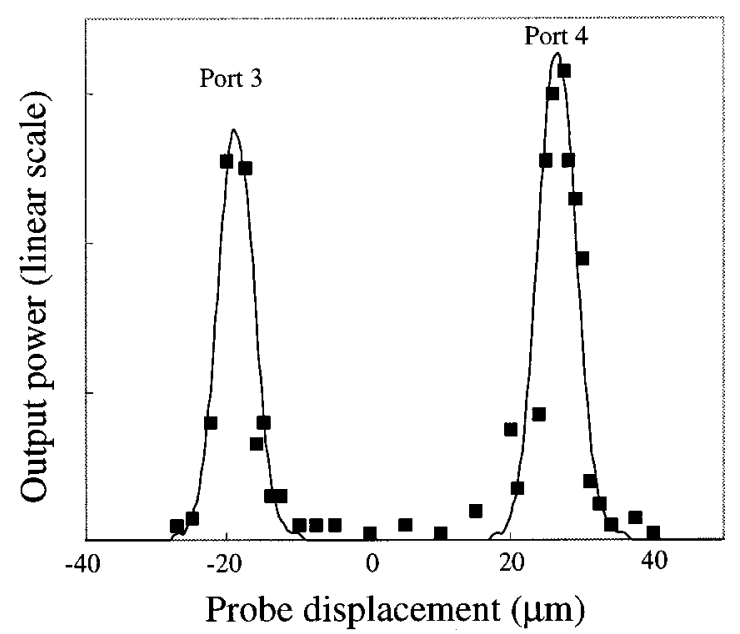

FIG. 4. Measured output optical power vs the probe displacement in the horizontal direction for a $2 \times 2 \mathrm{GaN} / \mathrm{AlGaN}$ heterostructure optical waveguide coupler The input optical signal is launched at port 1 (as illustrated in Fig. 3). 


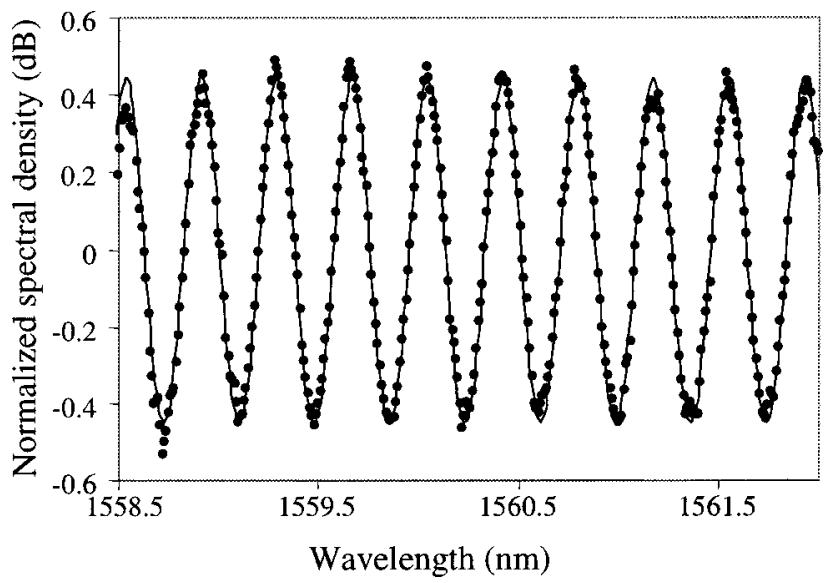

FIG. 5. Measured optical transmission spectrum (solid points) and numerical fitting using Eq. (3) (continuous line). Fitting parameters used: $n_{\text {eff }}$ $=2.315$ and $A=4.8 \mathrm{~dB}$.

Fresnel reflections of the two end facets of the waveguide. This excluded the uncertainties due to optical coupling efficiencies at the input and the output of the waveguide. An erbium-doped fiber amplifier (EDFA) without optical signal input was used in this measurement which provides a wideband amplified spontaneous emission (ASE). An optical spectrum analyzer was used to measure the ASE optical spectrum after it passes through the optical waveguide sample. The solid points in Fig. 5 show the measured optical spectrum which was normalized such that the average power spectral density is at $0 \mathrm{~dB}$. The continuous line in the same figure was calculated by using the normalized FP transmission equation:

$$
T(\lambda)=\left[1+R^{2} A^{2}-2 R A \cos \left(4 \pi L n_{\mathrm{eff}} / \lambda+\varphi_{0}\right)\right]^{-1},
$$

where $L=1.395 \mathrm{~mm}$ is the waveguide length, $R=\left[\left(n_{\text {core }}\right.\right.$ $\left.-1) /\left(n_{\text {core }}+1\right)\right]^{2}=16.02 \%$ is the power reflectivity of the waveguide end surface, $\varphi_{0}$ is an initial phase which can be determined by the best fitting between the measured and the calculated spectrum, and $A$ is the single-pass power attenuation through the waveguide. $n_{\text {eff }}$ is the effective index of the waveguide. Since the refractive indices for the core and the cladding are $n_{\text {core }}=2.335$ and $n_{\text {cladding }}=2.275$, respectively, with the waveguide geometry shown in Fig. 2, the effective index can be determined as $n_{\text {eff }}=2.315$ through a BPM simulation. To obtain the best fitting to the measured spectrum as shown in Fig. 5, $A=4.8 \mathrm{~dB}$ was used. Giving the waveguide length of $1.395 \mathrm{~mm}$, this gives a waveguide loss coefficient of $\sim 34.4 \mathrm{~dB} / \mathrm{cm}$. It is worth mentioning that in sample preparation, the cleaved waveguide end facets are not ideally flat nor are they exactly perpendicular to the waveguide axis, therefore, the effective facet reflectivity is less than $16.02 \%$. Due to this reason we expect that the actual attenuation of the waveguide should be significantly less than $4.8 \mathrm{~dB}$. Other optical loss mechanisms such as scattering at the waveguide edge can be further reduced by improving the etching process.

So far, the most popular application of III-nitride semiconductor materials is light emission in UV/blue wavelength regions. Their applications for optical emission in infrared have been investigated by exploring the effect of intersub- band transitions in GaN/AlGaN multiple quantum well structures. ${ }^{10}$ Here we would like to point out another possible application of III nitrides for realizing switchable photonics integrated circuits. Optical emission in the GaN/AlGaN waveguide has been demonstrated in UV wavelength regions by carrier injection. However, in infrared, which is far from the material band gap, both optical gain and absorption in GaN/AlGaN waveguide are expected to be negligible. Therefore, the material is essentially transparent in the optical communication wavelength windows around $1550 \mathrm{~nm}$. On the other hand, the material refractive index is a function of carrier density, and therefore, through carrier injection, the refractive index can be modulated. This index-controllable nature of $\mathrm{GaN} / \mathrm{AlGaN}$ heterostructures waveguide makes them an ideal candidate for optical phasors, ${ }^{11}$ such as MachZehnder modulators and AWGs. GaN has a much smaller refractive index $(n \approx 2.3)$ and potentially lower optical absorption compared to InP. In addition, carrier-induced refractive index change is independent of the state of signal polarization. This is superior to $\mathrm{LiNbO}_{3}$-based optical devices where polarization-dependent electro-optic effect is used. A switchable optical phasor array can be used as an optical switch or wavelength router. Because of the sub-nanosecond carrier lifetime in GaN and AlGaN (Ref. 12) and therefore the fast switching speed, optical packet switch will be made possible.

In summary, we have proposed the applications of IIInitride wide-band-gap semiconductors for fiber-optic communications in the infrared spectral region. We have demonstrated the feasibility of achieving functional waveguide optical devices using GaN/AlGaN heterostructures. In particular, a 3-dB-GaN/AlGaN heterostructures optical waveguide coupler has been designed, fabricated, and characterized. The possibility of developing photonic integrated circuits based on III-nitride wide-band-gap semiconductors for fiber-optical communications has been discussed.

This work was supported by the NSF through "UltraHigh-Capacity Optical Communications and Networks" (ECS-0123450) and DMR-0203373.

${ }^{1}$ C. Dragone, IEEE Photonics Technol. Lett. 3, 812 (1991).

${ }^{2}$ M. Okuno, K. Kato, Y. Ohmori, M. Kawachi, and T. Matsunaga, IEEE/ OSA J. Lightwave Technol. 12, 1597 (1994).

${ }^{3}$ N. Takato, T. Kominato, A. Sugita, K. Jingugi, H. Toba, and M. Kawachi, IEEE J. Sel. Areas Commun. 8, 1120 (1990).

${ }^{4}$ C. R. Dorr, C. H. Joyner, L. W. Stulz, and R. Monnard, IEEE Photonics Technol. Lett. 10, 117 (1998).

${ }^{5}$ InP-Based Materials and Devices, Physics and Technology, edited by O. Wada and H. Hasegawa (Wiley, New York, 1999), Sec. 2.4.

${ }^{6}$ The Blue Laser Diodes, edited by S. Nakamura and G. Fasol (Springer, Berlin, 1997).

${ }^{7}$ T. N. Oder, J. Y. Lin, and H. X. Jiang, Appl. Phys. Lett. 79, 2511 (2001).

${ }^{8}$ K. B. Nam, J. Li, M. L. Nakarmi, J. Y. Lin, and H. X. Jiang, Appl. Phys. Lett. 81, 1038 (2002).

${ }^{9}$ T. N. Oder, J. Y. Lin, and H. X. Jiang, Appl. Phys. Lett. 79, 12 (2001).

${ }^{10}$ C. Gmachl, S. V. Frolov, H. M. Ng, S. G. Chu, and A. Y. Cho, Electron. Lett. 37, 378 (2001).

${ }^{11}$ M. K. Smit and C. van Dam, IEEE J. Sel. Top. Quantum Electron. 2, 236 (1996).

${ }^{12}$ H. X. Jiang and J. Y. Lin, in III-Nitride Semiconductors Optical Properties I, edited by M. O. Manasreh and H. X. Jiang (Taylor and Francis, New York, 2002), p. 9. 